Fátima Oliveira (D)

Universidade do Porto

moliv@letras.up.pt

Fátima Silva (D)

Universidade do Porto

mhenri@letras.up.pt

\title{
O uso do Pretérito Imperfeito e do Pretérito Perfeito do Indicativo em português europeu por estudantes com cantonês como L1
}

\section{Resumo:}

Este trabalho tem como objetivo averiguar que condições de uso implicam mais desvios relativamente ao emprego do Pretérito Perfeito Simples (PPS) e do Pretérito Imperfeito (PI) por aprendentes chineses de PLE, de nível A2 (QECRL), com L1 cantonês, procurando encontrar algumas explicações para a sua ocorrência. A análise, quantitativa e qualitativa, parte do contraste entre o português e o cantonês quanto à marcação do tempo e do aspeto e descreve os desvios mais frequentes num corpus de produções escritas dos estudantes. Face a alguns resultados inesperados, procedeu-se também a uma verificação de ocorrências do Presente em vez do PPS e do PI, como forma de confirmar ou não, possíveis explicações. Os resultados indicam que não parece haver uma transferência direta do valor dos marcadores aspetuais perfetivos e imperfetivos do cantonês para a utilização do PPS e do PI ou do Presente, mas uma transferência da concetualização perfetivo/imperfetivo no cantonês, independentemente do tempo.

Palavras chave: Pretérito Imperfeito, Pretérito Perfeito Simples, L2, produção escrita, análise de desvios 


\begin{abstract}
:
The Use of Pretérito Imperfeito and Pretérito Perfeito in European Portuguese by L1 Cantonese Students

This work aims to verify what conditions of use imply more deviations from the use of Pretérito Perfeito Simples (PPS) and Pretérito Imperfeito (PI) by 21 Chinese learners of Portuguese, A2 level (QECRL), with Cantonese L1, and tries to find some explanations for its occurrence. The quantitative and qualitative analysis is based on the contrast between Portuguese and Cantonese in terms of tense and aspect and describes the most frequent deviations in a corpus of students' written productions. In face of some unexpected results, an analysis of the occurrences in which the Present occurs instead of the expected PPS and the PI was also performed, as a way of confirming or not, possible explanations. The results indicate that there does not appear to be a direct transfer of the value of the perfect and imperfect aspect markers of Cantonese to the use of PPS and PI or the Present, but a transfer of the perfect / imperfect conceptualization in Cantonese, regardless of the tense.
\end{abstract}

Keywords: Pretérito Imperfeito, Pretérito Perfeito Simples, L2, written productions, deviation analysis

\title{
Introdução
}

A distinção entre Pretérito Imperfeito (PI) e Pretérito Perfeito (PPS) do Indicativo em Português Europeu (PE) é tipicamente fonte de dificuldades para estudantes de PE, sobretudo para aqueles cujas línguas maternas não apresentam tal distinção. Um desses casos pode ser exemplificado por estudantes de português cuja L1 é o cantonês.

De um modo geral, podemos dizer que, enquanto o PE apresenta flexão verbal temporal, o cantonês é uma língua sem tempo verbal sendo esta informação veiculada por advérbios ou pelo contexto. Por outro lado, o PE não marca formalmente o aspeto, enquanto o cantonês apresenta vários marcadores aspetuais (cf. Oliveira, 2003, 2013; Sybesma, 2004).

Assim, tendo em conta esta disparidade entre as duas línguas, o objetivo deste estudo consiste em averiguar que condições de uso implicam mais desvios relativamente ao emprego do PPS e do PI por parte de aprendentes chineses de PLE, de nível A2 (Quadro Europeu Comum de Referência para as Línguas - QECRL, 2001), com 
L1 cantonês, procurando encontrar algumas explicações para a sua ocorrência.

Deste modo, expomos, na secção 2, algumas caraterísticas do PE em contraste com o cantonês relativamente à marcação do tempo e do aspeto. Na secção 3, apresentamos o estudo, no contexto do qual explicitamos o corpus de análise e a metodologia usada, analisamos os dados, de forma quantitativa e qualitativa, e discutimos os resultados obtidos. Na secção 4, apresentamos algumas observações finais.

\section{O português em contraste com o cantonês}

Tendo em conta as observações feitas acima sobre o comportamento das duas línguas relativamente à marcação do tempo e do aspeto, apresentamos de seguida, de forma sucinta, algumas caraterísticas destas línguas sobre esta questão, referindo, em primeiro lugar, alguns aspetos sobre a interpretação do PPS e do PI em PE e, em segundo lugar, algumas formas de marcar tempo e aspeto em cantonês.

\section{Algumas caraterísticas do PPS e do PI em PE}

De um modo geral, o tempo está associado à localização da situação, quer relativamente ao tempo da enunciação, quer a outro tempo marcado linguisticamente. $\mathrm{O}$ aspeto, por sua vez, diz respeito à estrutura temporal interna da situação, sendo relevantes distinções entre presença ou ausência de dinamismo, de telicidade ou de duração, entre outras.

Em línguas como o PE, alguns tempos verbais podem veicular tanto informações temporais como aspetuais, dependendo das classes aspetuais com as quais se combinam. O PPS dá fundamentalmente informação temporal de passado, não operando alterações aspetuais, ao combinar-se com qualquer classe aspetual. Pelo contrário, o PI pode dar informação temporal, mas pode alterar a classe aspetual de base, funcionando como um operador aspetual, como é o caso, a título exemplificativo, da transformação de eventos como fumar, em estados, a Maria fumava, que passa a ser um estado habitual, pelo efeito do PI. 
De seguida, apresentamos de modo muito breve algumas caraterísticas destes tempos, quer em frases simples, quer em frases complexas, tendo em atenção as possíveis combinações.

\section{Pretérito Perfeito}

\section{PPS em frases simples}

O PPS veicula informação temporal de anterioridade relativamente ao tempo da enunciação ou em relação a outro tempo expresso linguisticamente. Quanto ao aspeto, o PPS dá sistematicamente a informação de que a situação está terminada, não alterando a classe aspetual. Estas caraterísticas têm como consequências: i) poder ocorrer com todas as classes aspetuais básicas sem as alterar; ii) dar informação de terminado em todos os casos; iii) poder ocorrer em frases simples com todas as classes aspetuais ${ }^{1}$, como se pode ver no exemplo (1), com eventos, e em (2), com estados.

(1) A Ana caiu /leu a carta/ dançou /tossiu.

(2) A Ana esteve doente / foi simpática.

\section{PPS em algumas frases complexas}

Em frases complexas, embora o PPS possa associar-se com vários tempos verbais, neste estudo concentramo-nos apenas na combinação do PPS quer com o PPS, quer com o PI.

As frases complexas abordadas são apenas temporais com quando e alguns casos de completivas selecionadas por verbo dado que a articulação dos tempos pode ser diferente.

Assim, nos exemplos seguintes, com uma oração temporal com quando, a combinação de dois PPS em que a classe aspetual nas duas orações é um estado parece ser de difícil aceitação ${ }^{2}$ (cf. (3)). Relativamente ao exemplo (4), todas as frases são aceitáveis, na medida em

${ }^{1}$ Para a tipologia das classes aspetuais, cf. Oliveira (2003) e Cunha (2013).

${ }^{2}$ Note-se que, num exemplo como (3), se o estado (faseável) da temporal passar a evento, a combinação dos dois PPS torna-se possível:

A Rita foi simpática quando o Rui a convidou. 
que, em todos os casos, quer na oração principal, quer na temporal, ocorrem eventos, de classes aspetuais diferentes, no PPS.

(3)?? A Rita foi simpática /\#³ foi de Lisboa quando esteve bem-disposta.

(4) A Rita nadou/ bebeu um café/ saiu quando o Rui a convidou.

Nas frases completivas, a combinação entre as diferentes classes aspetuais apenas não pode ocorrer quando temos um predicado de indivíduo não faseável ${ }^{4}$ (cf. (6)).

(5) A Rita disse que o Rui foi simpático.

(6) \#A Rita disse que o Rui foi de Lisboa.

(7) A Rita disse que o Rui nadou/bebeu um café/caiu/esteve doente/espirrou.

\section{Pretérito Imperfeito}

\section{PI em frases simples}

No que se refere ao PI, embora possa localizar situações no passado, raramente o faz em relação ao tempo da enunciação, na medida em que é tipicamente um tempo anafórico, isto é, dependente de outro tempo expresso linguisticamente. Por ser também um tempo alargado, confere duração às situações e, por isso, pode aspetualmente transformar eventos em estados. Estas caraterísticas têm como consequências: i) poder ocorrer com eventos, transformando-os em estados [cf. (9)], e poder ocorrer com estados não os alterando [cf. (8)]; ii) só poder ocorrer em frases simples com alguns estados [cf. (8) vs (10)] ou quando operar alterações aspetuais transformando um evento em estado habitual [cf. (9)].

${ }^{3}$ Usa-se o símbolo '\#' para assinalar que se trata de uma frase não interpretável do ponto de vista semântico, mesmo se do ponto de vista sintático está bem construída.

${ }^{4}$ Para a distinção entre predicado de indivíduo e predicado de fase ('I-level predicate' e 'S-level predicate'), veja-se Carlson (1977). Para a distinção entre estados faseáveis e estados não faseáveis, veja-se Cunha (1998, 2004). 
(8) A Rita era simpática / era de Lisboa.

(9) A Rita nadava na piscina de Leça/ bebia um café \# (ao pequeno almo-

ço) /saía \# (ao fim de semana).

(10) ?? A Rita estava doente.

\section{PI em algumas frases complexas}

Tendo em conta que o PI em PE raramente pode ocorrer em frases simples, a não ser com predicados de indivíduo e quando é um estado habitual derivado, torna-se relevante apresentar de forma breve algumas combinações possíveis do PI com PPS e com PI em frases complexas temporais com quando e, também, alguns casos de frases completivas.

Em frases temporais com quando, o PI pode combinar-se com o PPS na ordem PI + PPS, com algumas restrições, em particular quando nas duas frases ocorrem estados e o PI se combina com predicados de indivíduo [cf. (11)]. No entanto, quando os predicados com os quais o PI se combina são predicados de fase, quer estados [cf. (13)], quer eventos [cf. (12)], esta configuração é possível, mas tendo como consequência que as orações que apresentam PI são transformadas em estados 5 .

(11) \# A Rita era simpática /\#era de Lisboa quando esteve bem-disposta.

(12) A Rita nadava /bebia um café /saía/espirrava quando o Rui a chamou.

(13) A Rita estava doente quando o Rui a visitou.

Quando, nas mesmas configurações das frases anteriores, o PI se combina com PI, verificam-se algumas alterações, como os exemplos (14)-(17) mostram. Em (14), a versão com era de Lisboa continua a não ser aceitável, dado que este predicado, sendo um predicado de indivíduo, é também um estado não faseável, não permitindo que, na combinação com a temporal, tenha uma transformação em estado habitual. Pelo contrário, na versão com ser simpático, embora sendo um predicado de indivíduo, é um estado faseável, permitindo, por isso, a repetição da situação. Na maior parte dos outros exemplos em que

${ }^{5}$ Esta transformação do PI em estados é já referida em Kamp \& Rohrer (1983), para o francês, e em Oliveira (1990), para o português. 
o PI, na oração principal, se combina com eventos [cf. (15)], a leitura apresentada para os estados faseáveis também se aplica [cf. (16)]. No entanto, em (17), a combinação de um predicado de fase, que é um estado não faseável, torna a combinação de dois PI de difícil aceitação.

(14) A Rita era simpática /\# era de Lisboa quando estava bem-disposta.

(15) A Rita nadava /bebia um café /saía quando o Rui a convidava.

(16) A Rita vivia em Paris quando frequentava a universidade.

(17) ??A Rita estava doente quando o Rui viajava.

Em frases completivas, o PI também se pode combinar com o PPS. No caso destas construções, contrariamente ao que acontece com as frases com quando, é a oração principal que serve de ponto de referência temporal. Assim, em (18), em que o PI se combina com estados, temos uma relação de subordinação temporal entre as duas orações. Em (19), em que o PI se combina com eventos, a única possibilidade de interpretação é a de que o PI transforma os eventos em estados habituais ou força, de alguma maneira, a repetição da situação, como é o caso de o Rui nadava, com leitura de estado habitual. Nos outros dois casos, estão disponíveis duas leituras desde que estejam presentes adjuntos temporais. Assim, o PI pode ter uma leitura de futuro do passado, se o adjunto escolhido for, respetivamente, amanhã ou em breve, havendo por isso subordinação temporal; se o adjunto adverbial selecionado for ao almoço ou à noite, temos uma leitura de repetição, que permite uma interpretação de habitualidade.

(18) A Rita disse que o Rui era simpático/ era de Lisboa/ estava doente.

(19) A Rita disse que o Rui nadava/ bebia um café (ao almoço/ amanhã) /saía (à noite/ em breve).

Estas diferentes leituras das combinações entre estes dois tempos revelam que é crucial conhecer distinções aspetuais básicas e alguns fatores que permitem a derivação aspetual para compreender diferenças entre estes dois tempos em PE. 


\section{Breves observações sobre o cantonês}

O cantonês, que faz parte do grupo das línguas siníticas, é uma língua tipologicamente muito distinta do português. Assim, no que diz respeito à marcação temporal, considera-se, em geral, que as línguas siníticas não têm marcação de tempo verbal [cf. Matthews, Yip, 1994; Sybesma, 2004; Sze-Wing, Siu-Pong, 2014, e.o.), na medida em que não têm meios morfológicos/semilexicais ou gramatical(izados) para marcar eventos no passado, sendo a marcação do tempo feita através de adverbiais temporais ou meramente determinada pelo contexto. Para ilustrar o que acabamos de dizer, vejam-se os seguintes exemplos de Sybesma (2004: 169).

$$
\begin{aligned}
& \text { (20) a. } \text { ngo }^{5} \quad\left(\mathrm{kam}^{4}-\mathrm{jat}^{6}\right) \quad \text { maai }^{5} \quad \mathrm{jat}^{1}-\mathrm{bun}^{2} \quad \mathrm{syu}^{1} \quad \mathrm{ge}^{3}-\mathrm{si}^{4}-\mathrm{hau}^{6} \\
& \text { 'quando eu estava a comprar um livro ontem...' } \\
& \text { b. } \mathrm{keoi}^{5} \mathrm{ji}^{5}-\mathrm{cin}^{4} \text { hai }{ }^{2} \text { Rotterdam } \mathrm{zyu}^{6} \\
& 3 \mathrm{~s} \text { before at Rotterdam live } \\
& \text { 'ele(a) costumava viver em Roterdão' } \\
& \text { c. } \text { keoi }^{5} \text { hai }^{2} \text { Rotterdam } \text { zyu }^{6} \\
& \text { 3s at Rotterdam live } \\
& \text { 'ele(a) vive em Roterdão' }
\end{aligned}
$$$$
\text { 1s yesterday buy one-CL book while }
$$

Em (20a), o exemplo é construído com um evento e a informação temporal é de passado, sem qualquer marca associada ao verbo, mas com um advérbio temporal. Em (20b), a informação temporal continua a ser de passado, mas o exemplo é construído com um estado e um adverbial temporal. Por último, (20c) é apresentado como uma situação atual, sem recurso a qualquer adverbial, sendo também construída com um estado.

No entanto, em cantonês, a marcação aspetual é bastante rica, apresentando marcadores para aspeto perfetivo, aspeto progressivo, aspeto contínuo, aspeto habitual, aspeto experiencial, entre outros (Chan, 2000; Matthews, Yip, 1994, e.o). 
Assim, quanto à perfetividade, apresenta tipicamente dois marcadores $-z O^{2}$ e $g w o^{1}-$, distinguindo-se entre si por $z O^{2}$ apresentar a situação como um todo fechado, terminativo [cf. (21)-(22)], e gwo ${ }^{l}$ sugerir experiência e uma situação que aconteceu pelo menos uma vez antes [cf. (23)-(24)] (Sybesma, 2004; Yip, Matthews, 2000; Lei, Lee, 2017).

(21) ngo $^{5}$ maai $^{5}-\mathrm{zo}^{2} \quad$ jat $^{1}-$ bun $^{2} \quad$ syu $^{1} \quad$ (Sybesma, $2004: 172$ ) $1 \mathrm{~s}$ buy- $\mathrm{ZO}^{2}$ one-CL book

'Eu comprei um livro'

(22) ngo5 kam4-jat6 maai5-zo2 jat1-bun2 syu1

1 s yesterday buy-ZO2 one-CL book

'Eu comprei um livro ontem'

(23) Léih heui-gwo Taai-hūng Gwún meih a? (Yip, Matthews, 2000: 94) 'Have you been to the Space Museum?'

(24) Ngóh meih yám-gwo nī jek jáu

'I've not drunk this wine before'

No que diz respeito à imperfetividade, esta é indicada pelos marcadores $g^{2}{ }^{2}$, progressivo, e $z y u^{6}$, contínuo (ongoing), que se distinguem por o primeiro se combinar com eventos e estados stage level, mas não com predicados individual level [cf. (25)-(27)] (Lam, 2009), e o segundo descrever uma atividade ou estado sem mudança [cf. (28)] (Yip, Matthews, 2000). Note-se que, em casos como (27), sem qualquer adverbial, é veiculada a informação de presente.

(25) Go-zan-si ni dung lau zung hei -gan. (Lam, 2009: 90) that CL time this CL flat still build GAN

'At that time this block of flats was still being built.'

(26) Camjat saam-dim-zong go zan, ngo zong zou-gan je (Lam, 2009: 91) yesterday three o'clock that time I still do GAN thing 'At three o'clock yesterday, I was still working.'

(27) Ngo jiga aamaam sik-gan je (Lam, 2009: 91)

I now just eat GAN thing

'I am just eating' 
(28) Gāan ūk deui-jyuh go hói. (Matthews \& Yip, 1994: 203) CL house face-CONT CL sea 'The house faces the sea.'

Sintetizando, $z \mathrm{O}^{2}$ é tipicamente um marcador de terminado, mas combina-se preferencialmente com eventos, sendo mais raro com estados ou podendo induzir leitura incoativa. Por seu turno, gan $^{l}$ é tipicamente um marcador de imperfetividade e combina-se com eventos e estados stage level.

Estes marcadores não indicam tempo. No entanto, parece haver a seguinte tendência: "perfective aspect is usually associated with the past and imperfective with no-past" (Huang, 1988: 125; Chan [et al.], 2004: 388). Acresce que estes marcadores aspetuais podem ocorrer sem adverbiais, mas, quando estes estão presentes, especificam quando a situação teve lugar no passado.

O marcador zero é compatível com estados e com eventos e, na ausência de qualquer adverbial temporal, ou alguma informação do contexto, a interpretação tende a ser a de Presente.

\section{Estudo}

Depois de uma breve descrição do comportamento do PPS e do PI em PE e de algumas observações sobre o cantonês, apresentamos, de seguida, o estudo realizado. Em primeiro lugar, descrevemos a metodologia adotada, em segundo, fazemos uma análise quantitativa e qualitativa dos dados e, finalmente, discutimos os resultados obtidos.

\section{Metodologia}

O estudo baseia-se em dados provenientes de produções escritas realizadas presencialmente em contexto letivo, essencialmente do tipo narrativo, tendo sido os temas propostos pelos docentes.

Cada estudante escreveu entre dois e três textos, de forma completamente autónoma, isto é, sem recurso a qualquer auxiliar, dicionário ou prontuário. No total, foram produzidos 78 textos, com uma média de 99 palavras cada um. 
O grupo de informantes era constituído por 21 estudantes, com idades compreendidas entre os 18 e 21 anos, provenientes de Macau e com o cantonês como L1, a frequentar um curso intensivo de português para estudantes universitários. Os dados foram coletados no nível A2, sendo o nível determinado através de um teste de posicionamento, aplicado no início do curso, com base nos descritores do QECRL (2001).

A metodologia seguida para análise dos dados é mista, isto é, quantitativa e qualitativa, e consistiu na recolha da amostra, identificação, descrição e explicação dos desvios [cf. Ellis, 1994, e.o.). Numa primeira fase, analisámos os dados relativos ao PPS e PI, tendo, posteriormente, incorporado os dados das ocorrências desviantes de Presente, nos casos em que o uso deste tempo substituía o PPS ou o PI, tempos esperados nos contextos em questão.

\section{Análise dos dados}

$\mathrm{Na}$ análise quantitativa, foi contabilizado o número total de ocorrências de PPS e PI nos textos, seguindo-se a identificação dos segmentos com desvios destes tempos, no contexto dos quais não são integrados desvios de flexão. A análise qualitativa, por sua vez, centrou-se sobretudo na descrição e explicação desses desvios.

\section{Análise quantitativa}

A tabela 1 resume os dados relativos às ocorrências do PPS e do PI, tendo em conta o número total de palavras, e os desvios produzidos pelos estudantes, incidindo naqueles que são mais frequentes. Tendo em consideração a percentagem de ocorrências no corpus dos dois tempos verbais, verificamos que o PPS ocorre menos vezes que o PI. No que se refere aos desvios, proporcionalmente, observa-se um número menor de desvios no uso do PI em relação aos desvios no emprego do PPS, pois os primeiros representam apenas cerca de 5\% da totalidade dos usos enquanto os segundos correspondem a cerca de $26 \%$. 
Tabela 1: ocorrências e desvios nos usos de PPS e PI

\begin{tabular}{|c|c|c|c|c|c|}
\hline $\begin{array}{c}\mathrm{N}^{\circ} \text { total } \\
\text { de pala- } \\
\text { vras }\end{array}$ & $\begin{array}{c}\mathrm{N}^{\mathrm{o}} \text { total de ocor- } \\
\text { rências PPS }\end{array}$ & $\begin{array}{c}\text { PPS des- } \\
\text { vios }\end{array}$ & $\%$ & $\begin{array}{c}\text { PPS em vez } \\
\text { de PI }\end{array}$ & $\%$ \\
\hline \multirow{3}{*}{7774} & 272 & 71 & 26,10 & 67 & 94,19 \\
\cline { 2 - 6 } & $\begin{array}{c}\mathrm{N}^{\circ} \text { total de ocor- } \\
\text { rências PI }\end{array}$ & PI desvios & $\%$ & $\begin{array}{c}\text { PI em vez de } \\
\text { PPS }\end{array}$ & $\%$ \\
\cline { 2 - 6 } & 436 & 22 & 5,04 & 13 & 59,90 \\
\hline
\end{tabular}

No que se refere aos desvios registados, há um tipo de desvio preponderante em cada uma das situações: no caso do PPS, em 94\% dos casos este tempo é usado em vez do PI; em relação ao PI, assinala-se também o domínio de desvios resultantes do uso do PI em vez do PPS, embora com uma percentagem menos significativa, cerca de $60 \%$.

Dada a representatividade destes tipos específicos de desvios, é sobre eles que incide a nossa análise.

Para além disso, tivemos ainda em consideração os resultados ponderados por estudante, calculando o desvio padrão tanto em relação ao uso total de cada um destes tempos como ao uso do PPS e do PI que apresentam desvios, tal como se pode ver no gráfico 1 .

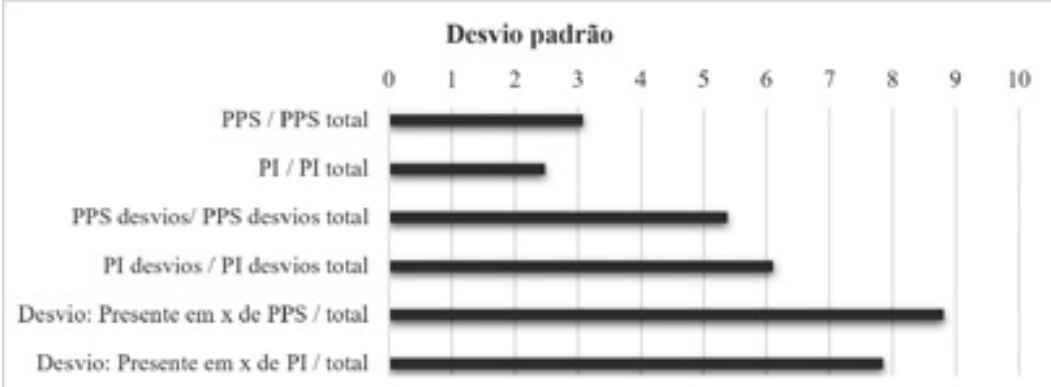

Gráfico 1: Desvio padrão no uso do PPS e do PI

A análise dos dados de cada estudante permite concluir que há uma maior variação no número total de ocorrências de PPS do que aquela 
que se verifica no emprego do PI. Quanto ao PPS, há estudantes que recorrem com frequência ao PPS, mas outros não, enquanto, no caso do PI, o número de ocorrências é mais aproximado entre os estudantes. No entanto, observa-se que os desvios no uso do PPS são mais consistentes entre os estudantes, pois há uma tendência para a mesma percentagem de desvios em relação ao uso total deste tempo, ao contrário do que acontece com o PI, em que há uma maior dispersão do número de desvios que cada estudante apresenta, embora não seja significativa.

\section{Análise qualitativa}

Depois de anotados e quantificados os desvios, analisámos os dados de modo a poder descrever os problemas detetados, tendo em conta, primeiro, as ocorrências de PPS em vez de PI e, depois, as do PI em vez de PPS, em frases simples, em frases complexas e em sequências de frases.

\section{Ocorrência de PPS em vez de PI}

Apresentam-se de seguida exemplos que ilustram as ocorrências destes desvios nos contextos referidos ${ }^{6}$.

\section{Frases simples}

(29) Esparguete com tomate e mariscos foi muito delicioso. (RES_14)

\section{Frases complexas}

(30) Pensei que o cozinheiro deveu pôr menos sal. (EXP_19)

\section{Sequências de frases}

(31) Quando eu era criança, eu costumava assistir à aula de piano aos sábados, eu detestei ir lá porque a professora parcia não concentrada quando me estava a ensinar tocar (...) (MEM_2)

Analisando os desvios produzidos em (29) - (31), verifica-se que a seleção do PPS em vez do PI ocorre em todos os casos com estados.

${ }^{6}$ Todos os exemplos retirados do corpus e devidamente identificados correspondem aos exemplos tal como foram produzidos pelos estudantes. 


\section{Ocorrência de PI em vez de PPS}

\section{Frases simples}

(32) As minhas férias de sonho eram na Itália com as minhas amigas. (FER_14)

\section{Frases complexas}

(33) Tinha um peixe, alimentava frequentemente, contudo, perdi-o quando mudávamos a casa. (MEM_3)

\section{Sequência de frases}

(34) Uma vez ele dizia me se eu comia o peixe, ele ia levar me ao parque de diversões. Eu comia mas ainda não vamos! (MEM_18)

Considerando os desvios produzidos em (32) - (34), observamos que o uso do PI em vez do PPS ocorre principalmente com eventos.

\section{Discussão dos resultados}

Verificámos que a percentagem de desvios decorrentes da seleção de PPS em vez de PI é maior do que a percentagem de desvios da seleção do PI em vez do PPS [cf. estudos sobre aquisição de cantonês como L1: Chan 2000).

Observámos também que há uma tendência para associar PPS a estados e PI a eventos, o que é contrário ao que esperaríamos tendo em conta a semântica destes tempos em PE e algumas restrições relativamente aos marcadores em cantonês.

Em face deste resultado inesperado, analisámos o mesmo corpus quanto aos desvios do PPS e do PI relativamente ao Presente. A razão fundamental da escolha deste tempo é, por um lado, o uso que dele fazem os informantes, e, por outro, questões de natureza linguística. Com efeito, o Presente tem semelhanças com o PI dado que, como operador aspetual, quando ocorre com eventos os transforma em estados habituais ou tem associada, pelo menos, repetição de situações [cf. (35)]. Mas contrariamente ao PI, quando ocorre com estados, apresenta leitura temporal de presente real [cf. (36)] não sendo também um tempo anafórico. 
(35) A Ana nada/ bebe café/ sai (à noite)/ espirra (de manhã).

(36) A Ana é de Lisboa/ é simpática/ está doente.

Em frases complexas como as que foram consideradas, é possível a combinação de Presente com Presente, quer com eventos [cf. (37)], quer com estados [cf. (38)], embora os predicados de indivíduo não faseáveis não sejam admitidos nas frases com quando tal como acontece com o PI e o PPS. Nas completivas, podem ocorrer eventos e também estados [cf. (39)].

(37) A Ana nada/ bebe café/sai/ espirra quando está bom tempo.

(38) A Rita é simpática/ \#é de Lisboa quando está bem-disposta.

(39) A Rita pensa que o Rui nada/ bebe café/ sai/ espirra/ é simpática/ é de Lisboa.

No corpus, observámos que há 17 ocorrências em que o Presente substitui o PPS e 21 ocorrências em que substitui o PI, como se sintetiza no gráfico 2 .

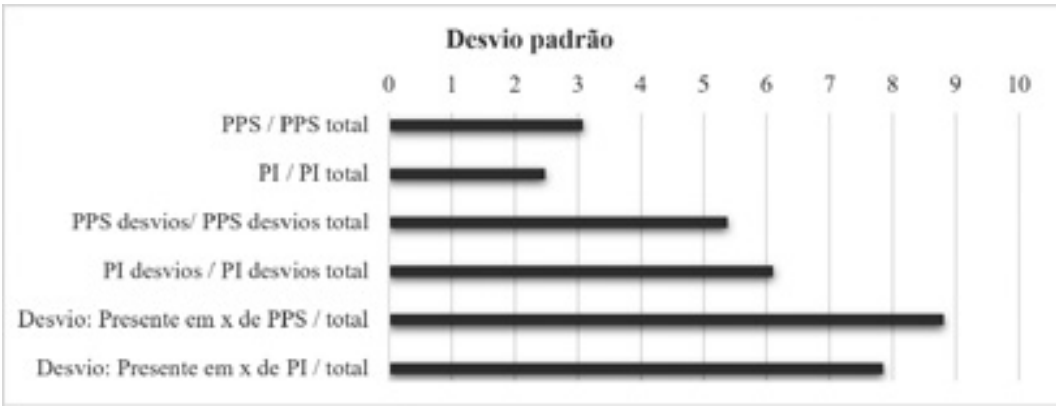

Gráfico 2: Desvios no uso do Presente

À semelhança da análise feita para os desvios do PPS e do PI, considerámos a ocorrência de Presente em vez de PPS e de Presente em vez de PI em frases simples, frases e complexas e sequências de frases [cf., respetivamente, exemplos (40)-(42) e (43)-(45)]. 


\section{Ocorrência de Presente em vez de PPS}

Frases simples

(40) Eu também faço as honras (...) (FER_17)

\section{Frases complexas}

(41) Eu fui num restaurante em China continentaç qie as amigas recomendam-me. (RES_17)

\section{Sequência de frases}

(42) $\mathrm{Na}$ verdade, não gostava muito de comer peixe. Porque que chava que o sabor do peixe é fedorento. Mas eu mudo da minha opinião por uma experiência. (EXP_20)

\section{Ocorrência de Presente em vez de PI}

\section{Frases simples}

(43) A minha primeira experiência que comi bacalhau foi num restaurante português em Macau. O prato é bacalhau cozido. (EXP_12)

\section{Frases complexas}

(44) Quando era criança, os meus pais tem de trabalhar. (MEM_13)

\section{Sequência de frases}

(45) Anteontem, eu fui ao restaurante ABC com a minha família. (...) A seguir, provei o prato de bacalhau que é delisioso. Infelizmente estava frio. Além disso, adoro sobremesas. (...) Por fim, provei o gelado de iogurte que é muito fresco. (RES_20)

Assim, em virtude destes resultados, relativamente ao contraste PPS/PI, é de salientar que tanto o PE como o cantonês têm meios para veicular o aspeto terminado, mas, enquanto o PE o faz através do PPS, o cantonês usa marcadores perfetivos. Quanto à imperfetividade, o PE pode recorrer a tempos verbais ou a construções perifrásticas (PI ou Presente e progressivo, p.e.) e o cantonês a 2 marcadores: progressivo e contínuo. Estes últimos marcadores, tendo em conta os estudos sobre o cantonês, aproximam-se do progressivo em PE, mas 
não necessariamente do PI ou do Presente, o que parece estar na base de dificuldades em compreender diferentes operações aspetuais que o PI ou o Presente podem fazer.

No que diz respeito ao uso do Presente em vez do PPS ou do PI, a sua ocorrência observa-se sobretudo em frases complexas e sequências de frases, evidenciando que uma das grandes dificuldades a este nível é a sequencialização de tempos, em particular a subordinação temporal.

O não domínio do PPS e do PI revela-se na dificuldade em considerar que os estados podem ocorrer tanto no PPS (o tempo preferido com estados pelos informantes) como também no PI.

Assim, não parece haver uma transferência do valor dos marcadores aspetuais perfetivos e imperfetivos do cantonês para a utilização do PPS e do PI, mas parece haver uma transferência da concetualização perfetivo/imperfetivo no cantonês, isto é, terminado vs ongoing, independentemente do tempo. Os desvios com o Presente, ao ocorrer sobretudo com estados, confirmam essa oposição.

\section{Considerações finais}

Neste trabalho, propusemo-nos analisar que condições de uso implicam mais desvios relativamente ao uso do PPS e do PI por parte de aprendentes chineses de PLE, de nível A2, com L1 cantonês.

Os resultados obtidos revelam que a distinção entre PPS e PI não está estabilizada no nível A2, o que se compreende, dado que o contraste entre estes dois tempos é trabalhado neste nível.

Os resultados e a sua discussão indiciam que é necessário ter em conta questões de aspeto, pelo menos a distinção entre estados e eventos, no ensino-aprendizagem do PPS e do PI, tendo em conta que os dados apontam para uma não transferência dos valores dos marcadores aspetuais perfetivos e imperfetivos do cantonês para a utilização dos tempos em PE. Consideramos ainda que é crucial uma consciencialização linguística para o facto de o PI raramente poder surgir em frases simples, dado que é na maioria dos casos um tempo anafórico, necessitando de estar normalmente associado a outro tempo, 
preferencialmente o pretérito perfeito, o que tem consequências na construção de frases complexas e também em sequências mais longas. Por estas razões, parece-nos também fundamental que as condições de sequencialização de tempos, com subordinação temporal ou não, sejam objeto de um trabalho pedagógico sistemático.

\section{Referências bibliográficas}

CARLSON, G. (1977), Reference to Kinds in English, Ph. D. dissertation, University of Massachusetts, Amherst.

CHAN, Y. L. E. (2000), Verb Semantics and Aspect in the Language of Cantonese-speaking Preschoolers, The University of Hong Kong, Hong Kong.

CHAN, Y. H., YAP, F. H., SHIRAI, Y., MATTHEWS, S. (2004), “A Perfective-imperfective Asymmetry in Language Processing: Evidence from Cantonese" em: Proceedings of the 9th International Symposium on Chinese Languages and Linguistics, Academia Sinica and the Graduate Institute of Linguistics, National Taiwan University, Taipei, pp. 383-391.

CONSELHO DA EUROPA (2001), Quadro Europeu Comum de Referência para as Línguas, Asa, Porto.

CUNHA, L. F. (1998), As construções com progressivo no português: uma abordagem semântica, Dissertação de Mestrado, FLUP, Porto.

CUNHA, L. F. (2004), Semântica das Predicações Estativas: para uma Caracterização Aspectual dos Estados, Tese de Doutoramento, FLUP, Porto (Lincom GmbH 2007).

CUNHA, L. F. (2013), “Aspeto” em: Raposo, E. [et al.] (eds.), Gramática do Português, FCG, Lisboa, pp. 585-622.

ELLIS, R. (1994), The Study of Second Language Acquisition, OUP, Oxford. KAMP, H., ROHRER, C. (1983), "Tense in texts” em: Bauerle, R., Schwarze, C., von Stechow, A. (eds.), Meaning, Use and Interpretation of Language, de Gruyter, Berlin, pp. 250-269.

HUANG, L. (1988), Aspect: A General System and its Manifestation in Mandarin Chinese, Student Book Co, Taipei.

LAM, C. F. (2009), “A Comparison between the Cantonese Aspect Markers gan and haidou", Rivista di Grammatica Generativa, 34, pp. 87-113. 
LEI, M. K., LEE, T. H. (2017), "Early Knowledge of the Interaction between Aspect and Quantification: Evidence from Child Cantonese" em: LaMendola, M., Scott, J. (eds.). Proceedings of the 41st Annual Boston University Conference on Language Development, Cascadilla, Somerville-MA, pp. 424-435.

MATTHEWS, S., YIP, V. (1994), Cantonese: A Comprehensive Grammar, Routledge, London.

OLIVEIRA, F. (1990), "Funções discursivas de alguns tempos do Passado em Português" em: Mateus M. H. M., Lopes, O. (eds.), Encontro de Homenagem a Óscar Lopes, Fundação Eng. António Almeida, Porto, pp. 165-186. OLIVEIRA, F. (2003), “Tempo e Aspecto” em: Mateus, M. H. M. [et al.], Gramática da Língua Portuguesa, Caminho, Lisboa, pp. 127-178.

OLIVEIRA, F. (2004), "O Imperfeito e o Tempo dos Indivíduos" em: Oliveira, F., Duarte, I. M. (eds.), Da Língua e do Discurso, Campo das Letras, Porto, pp. 505-528.

OLIVEIRA, F. (2013), “Tempo verbal” em: Raposo, E. [et al.] (eds.), Gramática do Português, FCG, Lisboa, pp. 509-556.

SZE-WING, T., SIU-PONG, C. (2014), "Aspects of Cantonese Grammar" em: Huang, J., LI, Y.-H. A., SIMPSON, A. (eds.), The Handbook of Chinese Linguistics, Wiley, London, pp. 601-628.

SYBESMA, R. (2004), "Exploring Cantonese Tense" em: Cornips, L., Doetjes, J., Linguistics in the Netherlands, John Benjamins, Amsterdam, pp. 169-180.

YIP, V., MATTHEWS, S. (2000), Intermediate Cantonese: A Grammar and Workbook, Routledge, London. 